\title{
Microtubule associated proteins and motors required for ectopic microtubule array formation in S. cerevisiae
}

Brianna R. King ${ }^{*}$, Janet B. Meehl†, Tamira Vojnar, Mark Wineył, Eric G. Muller*, and Trisha N. Davis*1

* Department of Biochemistry, University of Washington, Seattle, WA 98195

† Department of Molecular Cellular and Developmental Biology, University of Colorado, Boulder, CO 80309

$\ddagger$ Department of Molecular and Cellular Biology, University of California, Davis, CA 95616 
bioRxiv preprint doi: https://doi.org/10.1101/2021.01.12.426450; this version posted January 13,2021 . The copyright holder for this preprint

(which was not certified by peer review) is the author/funder, who has granted bioRxiv a license to display the preprint in perpetuity. It is made available under aCC-BY-NC-ND 4.0 International license.

Running title: S. cerevisiae MAPs in MT array formation

Key words: microtubule nucleation, microtubule organization, microtubule associated proteins, motors

${ }^{1}$ Corresponding author: Department of Biochemistry, University of Washington, UW Box 357350, 1705 NE Pacific St, Seattle, WA 98195. Email: tdavis@uw.edu 


\begin{abstract}
The mitotic spindle is resilient to perturbation due to the concerted, and sometimes redundant, action of motors and microtubule-associated proteins. Here we utilize an inducible ectopic microtubule nucleation site in the nucleus of Saccharomyces cerevisiae to study three necessary steps in the formation of a bipolar array: the recruitment of the $\gamma$-tubulin complex, nucleation and elongation of microtubules, and the organization of microtubules relative to each other. This novel tool, an Spc110 chimera, reveals previously unreported roles of the microtubule-associated proteins Stu2, Bim1, and Bik1, and the motors Vik1 and Kip3. We report that Stu2 and Bim1 are required for nucleation and that Bik1 and Kip3 promote nucleation at the ectopic site. Stu2, Bim1, and Kip3 join their homologs XMAP215, EB1 and kinesin-8 as promoters of microtubule nucleation, while Bik1 promotes MT nucleation indirectly via its role in SPB positioning. Further, we find that the nucleation activity of Stu2 in vivo correlates with its polymerase activity in vitro. Finally, we provide the first evidence that Vik1, a subunit of Kar3/Vik1 kinesin-14, promotes microtubule minus end focusing at the ectopic site.
\end{abstract}

\title{
Introduction
}

High fidelity distribution of genetic material during mitosis requires a dramatic reorganization of microtubules (MTs): the transition from the radiating network of MTs during interphase to the tightly localized bipolar spindle. To form this ordered mitotic array, nucleation of new MTs must be initiated and controlled, and existing MTs must be re-organized.

Successful spindle assembly requires the concerted action of MT organizing centers (MTOCs), motors, and MT associated proteins (MAPs). MTOCs define the distal ends of the spindle and position the spindle in the cell. Motors and MAPs, on the other hand, bind to the MT lattice and have a large variety of functions, from regulating the dynamic instability of MTs to facilitating MT transport. 
Several factors complicate our understanding of the roles that MAPs play. First, MAPs act en masse on the multi-micrometer long spindle; many copies of a single protein or a protein complex work together to perform their function efficiently (Page et al. 1994; Geiser et al. 1997; Miller et al. 1998; Miller et al. 2000; Derr et al. 2012; Furuta et al. 2013). Second, MAPs can function either redundantly (Hoyt et al. 1992; Roof et al. 1992; O'Connell et al. 1993; DeZwaan et al. 1997; Cottingham et al. 1999) or antagonistically (O'Connell et al. 1993; Saunders et al. 1997). The concerted MAP interactions result in a bipolar spindle that is resilient to perturbation. While this resiliency is advantageous for life, the interplay among MAPs makes assigning roles to individual MAPs difficult. Therefore, we utilized a tool that promotes MT array formation at a site that is ectopic to the bipolar spindle-an inducible Spc110 chimera $(\operatorname{Spc110c}(T))($ Figure 1). Spc110 is a key component of the yeast spindle pole body (SPB) that recruits the conserved $\gamma$-tubulin complex to the poles. Using the chimerabased assay, we have measured the contribution of five highly conserved families of MAPs and motors to MT array nucleation and organization. The tumor-overexpressed gene (TOG) polymerase Stu2, the end-binding (EB) protein Bim1, the CLIP170 homolog Bik1, and the kinesin-8 and kinesin-14 motor proteins, Kip3 and Vik1, were examined. Each have been previously implicated in MT nucleation and spindle assembly.

Saccharomyces cerevisae has a closed mitosis, and nucleation of new MTs during spindle assembly is narrowly localized to the nuclear face of the SPB. Of the five candidate budding yeast proteins, four have homologs that are implicated in MT nucleation in other organisms. Though these proteins primarily bind the MT lattice, four interact with components of the SPB: Stu2, Bim1, Bik1, and Vik1 (Wigge et al. 1998; 
Manning et al. 1999; Newman et al. 2000; Usui et al. 2003; Wang et al. 2012).

Furthermore, homologs of Stu2, Bim1, and Kip3 were shown to promote MT nucleation both in vivo and in vitro (Popov et al. 2002; Vitre et al. 2008; West and McIntosh 2008; Erent et al. 2012; Wieczorek et al. 2015; Thawani et al. 2018), and Stu2 specifically promotes cytoplasmic MT nucleation (Gunzelmann et al. 2018). Conversely, Plk1, the fission yeast kinesin-14 motor, suppresses MT nucleation (Olmsted et al. 2014).

Spindle assembly also requires careful regulation of dynamic instability at the MT plus ends, which extend away from the SPB and connect to the kinetochores. In the case of Stu2, Bim1, and Bik1, there is evidence of MT stabilization for each. Stu2 is a member of the XMAP215 family of MT polymerases that promote growth rates (Brouhard et al. 2008). Bim1 inhibits catastrophe and does so in complex with Bik1 (Blake-Hodek et al. 2010). Kip3, on the other hand, destabilizes long MTs and stabilizes short MTs (Gupta et al. 2006; Varga et al. 2006; Tischer et al. 2009; Gardner et al. 2011; Reese et al. 2011; Su et al. 2011; Melbinger et al. 2012; Fukuda et al. 2014).

In addition to regulating the length of MTs, MAPs or motors can function to organize MTs relative to each other. Specifically, interpolar MTs are organized to overlap in the midzone, while kinetochore MTs are focused toward MTOCs via their minus ends in order to generate tension. Dynein and kinesin-14 motors promote MT minus end focusing at the MTOCs in D. melanogaster and H. sapiens (Endow and Komma 1998; Kwon et al. 2008; Lecland and Lüders 2014). No such activity has been reported in budding yeast; however mutations that weaken the nuclear SPB-MT 
interface are synthetically lethal with Kar3 and Vik1 (of the Kar3Vik1 complex)

(Greenland et al. 2010).

\section{Materials and Methods}

\section{Media}

YPD was prepared as described (Burke et al. 2000). YPD 3x ADE media is YPD with 5 $\mathrm{mg} / \mathrm{ml}$ adenine. LoFlo S liquid medium contains $1.93 \mathrm{~g} / \mathrm{l}$ yeast nitrogen base LoFlo (Formedium ${ }^{\mathrm{TM}}$ ) plus $5 \mathrm{~g} / \mathrm{l}$ ammonium sulfate. LoFlo $\mathrm{SD}^{+}$medium is LoFlo $\mathrm{S}$ medium with $2 \%$ glucose and 1/100 dilution of a filter sterilized solution of L-amino acids and nutrients containing $0.2 \%$ arginine, histidine and methionine, $0.3 \%$ isoleucine, leucine, and lysine, $0.4 \%$ tryptophan, $0.5 \%$ phenylalanine and threonine, $0.25 \%$ uracil and $1.5 \%$ adenine. The high adenine suppressed the formation of red fluorescent pigment in our ade2, ADE3 strains.

\section{Strain construction}

All strains used in this study are derivatives of W303 (Thomas and Rothstein 1989) (Table S1). Transformations were performed by a standard lithium acetate method (Gietz et al. 1995). The tetrameric Spc110 chimera construct, Spc110c(T), consisting of SPC110(1-220a.a.)-GCN4-GFP-lacl was integrated at ADE2 with a promoter specific to the transcription factor $Z_{4} E V$ as previously described (Lyon et al., 2016). Z4EV was integrated at CAN1 with a $\beta$-estradiol inducible promoter (Mclsaac et al., 2013). 256 copies of a lacO repeat were integrated into chromosome XII as described previously (Lyon et al., 2016). MAPs were C-terminally tagged with 3 V5 epitopes and the auxinresponsive protein IAA7 (Shetty et al. 2019). The ubiquitin ligase complex protein OsTIR1, necessary for auxin-inducible degradation, was integrated at LEU2. Spc97 was C-terminally tagged with mCherry. GFP-Tub1 under the TUB1 promoter was integrated at URA3 using pAFS125 (Straight et al. 1997). 


\section{Electron tomography}

TVY11-123D was incubated with $1 \mathrm{mM}$ auxin (Millipore Sigma, St. Louis, MO) for 2.5 hours (Spc110-AID degradation greater than 90\%). Cells were collected on a Millipore filter by vacuum filtration and high pressure frozen using a Wohlwend Compact 02 high pressure freezer (Technotrade International, Manchester, New Hampshire). Cells were freeze substituted at low temperature in acetone containing $0.25 \%$ glutaraldehyde and $0.1 \%$ uranyl acetate, then infiltrated with Lowicryl HM20 resin. After polymerization, 350 $\mathrm{nm}$ serial sections were collected on copper slot grids coated with formvar, post-stained with $2 \%$ aqueous uranyl acetate and Reynold's lead citrate. Tilt series were collected on a Technai F30 microscope. Tomograms were computed and modeled using the IMOD software package (Kremer et al. 1996; Mastronarde 1997; O'Toole et al. 2002).

\section{$\underline{\gamma \text {-tubulin complex recruitment assay }}$}

Cells were grown asynchronously in YPD $3 x A D E$ at $25^{\circ} \mathrm{C}$ to $40 \mathrm{Klett}$ units, within log phase. Cells were first pulsed with $0.1 \mu \mathrm{M} \beta$-estradiol (Millipore Sigma) for 10 minutes and then washed with YPD 3xADE. Cells were then treated with $15 \mu \mathrm{g} / \mathrm{mL}$ nocodazole (Millipore Sigma) for 2.5 hours. In cells harboring a protein tagged with auxin-inducible degron, cells were simultaneously treated with $1 \mathrm{mM}$ auxin (Millipore Sigma) during the 2.5 hour incubation. Cells were pelleted and resuspended in $100 \mu \mathrm{l}$ of LoFlo SD medium, placed on Lo-Flo SD + 1\% agarose pad (SeaKem ${ }^{\circledR}$ Gold Agarose, Lonza, Rockland, ME) supplemented with $15 \mu \mathrm{g} / \mathrm{mL}$ nocodazole and $1 \mathrm{mM}$ auxin, as previously described (Muller et al., 2005) (https://www.youtube.com/watch?v=ZrZVbFg9NE8). Cells were imaged using either a DeltaVision system (Applied Precision, Issaquah, WA) or an Axio Observer (Carl Zeiss). The DeltaVision system was equipped with IX70 inverted microscope (Olympus, Center Valley, PA), a Coolsnap HQ digital camera (Photometrics, Tucson, AZ), and a U Plan Apo 100X objective (1.35 NA). The inverted Axio Observer was equipped with an ORCA-Flash4.0 V3 Digital CMOS camera (Hamamatsu), a Spectra X LED light engine (Lumencor), a Plan-APOCHROMAT $63 X / 1.46$ objective (Zeiss), and a 432/523/702 nm BrightLine® triple-band bandpass filter (Semrock, Inc., Rochester, NY). 
For the recruitment assay, the $\operatorname{Spc} 110 \mathrm{c}(\mathrm{T})$ was visualized using its integrated GFP fluorescence and $\gamma$-tubulin complexes were visualized with Spc97-mCherry. GFP was imaged with $0.25 \mathrm{~s}$ exposures, mCherry was imaged with $0.4 \mathrm{~s}$ exposures, and 250.2 $\mu \mathrm{m} z$-sections were taken. Images taken on the DeltaVision microscope were binned 2 $\times 2$, while images taken on the Axio Observer were not binned.

Recruitment analysis was performed using the Spots colocalization function of Imaris software (Bitplane, Switzerland) (RRID: SCR_007370). Spc110(1-220a.a.)-GCN4-GFPlacl puncta were located by assigning an $X Y$ diameter of $0.4 \mu \mathrm{m}$ and a $Z$ diameter of 1.3 $\mu \mathrm{m}$. Spc97-mCherry puncta were located by assigning an $X Y$ diameter of $0.4 \mu \mathrm{m}$ and a $Z$ diameter of $1.8 \mu \mathrm{m}$. Quality of spots was manually determined. To exclude background fluorescence of the agarose pad in the total number of puncta, GFP and mCherry fluorescent puncta were first colocalized to be within $4 \mu \mathrm{m}$. These filtered spots were then colocalized within $0.5 \mu \mathrm{m}$ and reported as the percent of $\operatorname{Spc} 110 \mathrm{c}(\mathrm{T})$ puncta with recruitment events.

\section{MT formation assay}

Cells were grown asynchronously in YPD $3 \times A D E$ at $25^{\circ} \mathrm{C}$ to $40 \mathrm{Klett}$ units, within log phase. Cells were then treated with $15 \mu \mathrm{g} / \mathrm{mL}$ nocodazole for 1 hour. $1 \mathrm{mM}$ auxin was then added, and the incubation continued for an additional hour. $0.1 \mu \mathrm{M} \beta$-estradiol was then added, and the incubation continued for an additional 1.5 hours. Cells were released from nocodazole and $\beta$-estradiol and treated with only $1 \mathrm{mM}$ auxin for 10 minutes. Cells were pelleted and placed on Lo-Flo agarose pads supplemented with auxin. Cells were imaged at 10-minute intervals for 2.5 hours as described above for the $\gamma$-tubulin recruitment assay.

For the MT formation assay, the Spc110c(T) and Spc97-mCherry were visualized and acquired as described for the recruitment assay, with MTs visualized using GFP-Tub1 and only $150.2 \mu \mathrm{m} z$-sections.

MT formation analysis was performed manually using Imaris software (Bitplane). 


\section{Calculations for an indirect effect of Bik1}

In control cells where SPBs move to the daughter, the fraction of those cells that form Spc110c(T)-associated MT arrays, or Adaughter, is 0.58 . When SPBs remain in the mother, the fraction, or Amother, is 0.083 . When Bik1-AID is degraded, the fraction of cells with SPBs remaining in the mother, or SPBmother, is 0.95 , while the fraction of cells with SPBs moving to the daughter, or SPBdaughter, is 0.048 . If the effect of Bik1-AID on ectopic MT array formation is due purely to SPB localization, the predicted fraction of cells with $\operatorname{Spc} 110 \mathrm{c}(\mathrm{T})$-associated MT arrays is 0.11 , as given by the following equation:

$$
\text { Predicted total fraction }=S P B_{\text {daughter }} \times A_{\text {daughter }}+S P B_{\text {mother }} \times A_{\text {mother }}
$$

\section{Line scan analysis}

Ten-pixel wide lines along Spc110c(T)-associated MT arrays were drawn manually using ImageJ (National Institutes of Health, United States), and measured values were the average fluorescence intensity at each point on the line scan. Background fluorescence was calculated using the average of the last five points of the line scan. After subtraction of background, line scans were analyzed using the peakfinder and trapz functions of Matlab (MathWorks, United States).

\section{Tables}

Table S1. Strains used in this study ${ }^{1}$.

\begin{tabular}{|l|l|l|}
\hline Strain name & Genotype & Established in \\
\hline TVY11-123D & MATa his3-11,15 trp1-1 & This study \\
& ADE2::Z4EVpr-SPC110(1-220)- & \\
& GCN4LI-GA-GFP-Lacl CAN1::NatMX- & \\
& ACT1pr-Z4EV ChrXII-R::IacO::TRP1. & \\
& SPC97-mCherry::hphMX LEU2::GFP- & \\
& Tub1 SPC110-AID:kanMX & \\
& URA3::OS-TIR1-myc3 & \\
\hline RKY44 & MATa/MAT $\alpha$ his3-11,15/his3-11/15 & This study \\
& leu2-3,112/leu2-3,112 trp1-1/trp1-1 & \\
& Ura3-1/ura3-1 & \\
\hline
\end{tabular}

\footnotetext{
${ }^{1}$ All strains contain the same markers as RKY44 except where noted.
} 


\begin{tabular}{|c|c|c|}
\hline & $\begin{array}{l}\text { ade2-1/ADE2::Z4EVpr-SPC110(1- } \\
\text { 220)-GCN4LI-GA-GFP-Lacl } \\
\text { can1-100/CAN1::NatMX-ACT1pr-Z4EV } \\
\text { ChrXII-R/ChrXII-R::IacO::TRP1 } \\
\text { SPC97-mCherry:::hphMX/SPC97- } \\
\text { mCherry::hphMX }\end{array}$ & \\
\hline RKY52 & TUB1/URA3::GFP-TUB1 & This study \\
\hline RKY174 & vik14::natMX/vik14::natMX & This study \\
\hline RKY31 & $\begin{array}{l}\text { vik14::natMX/vik14::natMX } \\
\text { TUB1/URA3::GFP-TUB1 }\end{array}$ & This study \\
\hline RKY56 & kip3 $\Delta:: H I S 3 M X 6 / k i p 3 \Delta:: H I S M X 6$ & This study \\
\hline RKY49 & $\begin{array}{l}\text { kip3 }:: H I S 3 M X 6 / k i p 3 \Delta:: H I S M X 6 \\
\text { TUB1/URA3::GFP-TUB1 }\end{array}$ & This study \\
\hline RKY14 & $\begin{array}{l}\text { STU2-3V5-IAA7:kanMX/STU2-3V5- } \\
\text { IAA7:kanMX } \\
\text { LEU2::pDPD1-OsTIR1/LEU2:::pDPD1- } \\
\text { OsTIR1 } \\
\text { HIS3::NUF2- } \\
\text { mTurquoise2/HIS3::NUF2mTurquoise2 }\end{array}$ & This study \\
\hline RKY7 & $\begin{array}{l}\text { STU2-3V5-IAA7:kanMX/STU2-3V5- } \\
\text { IAA7:kanMX } \\
\text { LEU2:::pDPD1-OsTIR1/LEU2::pDPD1- } \\
\text { OsTIR1 } \\
\text { TUB1/URA3::GFP-TUB1 } \\
\text { HIS3::NUF2- } \\
\text { mTurquoise2/HIS3::NUF2mTurquoise2 }\end{array}$ & This study \\
\hline RKY47 & $\begin{array}{l}\text { BIM1-3HA-IAA7-kanMX6/BIM1-3HA- } \\
\text { IAA7-kanMX6 } \\
\text { LEU2:::pDPD1-OsTIR1/LEU2:::pDPD1- } \\
\text { OsTIR1 }\end{array}$ & This study \\
\hline RKY46 & $\begin{array}{l}\text { BIM1-3HA-IAA7::kanMX6/BIM1-3HA- } \\
\text { IAA7::kanMX6 } \\
\text { LEU2::pDPD1-OsTIR1/LEU2::pDPD1- } \\
\text { OsTIR1 } \\
\text { TUB1/URA3::GFP-TUB1 }\end{array}$ & This study \\
\hline RKY51 & $\begin{array}{l}\text { BIK1-3HA-IAA7::kanMX/BIK1-3HA- } \\
\text { IAA7::kanMX } \\
\text { LEU2::pDPD1-OsTIR1/LEU2::pDPD1- } \\
\text { OsTIR1 }\end{array}$ & This study \\
\hline RKY36 & $\begin{array}{l}\text { BIK1-3HA-IAA7::kanMX/BIK1-3HA- } \\
\text { IAA7::kanMX }\end{array}$ & This study \\
\hline
\end{tabular}




\begin{tabular}{|c|c|c|}
\hline & $\begin{array}{l}\text { LEU2::pDPD1-OsTIR1/LEU2::pDPD1- } \\
\text { OsTIR1 } \\
\text { TUB1/GFP-TUB1::URA3 }\end{array}$ & \\
\hline RKY122 & $\begin{array}{l}\text { STU2-3HA-IAA7:kanMX/STU2-3HA- } \\
\text { IAA7:kanMX } \\
\text { HIS3::pDPD1-OsTIR1/HIS3::pDPD1- } \\
\text { OsTIR1 } \\
\text { TUB1/URA3::GFP-TUB1 } \\
\text { LEU2::STU2p-STU2-3V5:LEU2/ } \\
\text { LEU2::STU2p-STU2-3V5:LEU2 }\end{array}$ & This study \\
\hline RKY126 & $\begin{array}{l}\text { STU2-3HA-IAA7:kanMX/STU2-3HA- } \\
\text { IAA7:kanMX } \\
\text { HIS3::pDPD1-OsTIR1/HIS3::pDPD1- } \\
\text { OsTIR1 } \\
\text { TUB1/URA3::GFP-TUB1 } \\
\text { LEU2::STU2p-STU2(R200A)- } \\
\text { 3V5:LEU2/ LEU2::STU2p- } \\
\text { STU2(R200A)-3V5:LEU2 }\end{array}$ & This study \\
\hline RKY133 & $\begin{array}{l}\text { STU2-3HA-IAA7:kanMX/STU2-3HA- } \\
\text { IAA7:kanMX } \\
\text { HIS3::pDPD1-OsTIR1/HIS3::pDPD1- } \\
\text { OsTIR1 } \\
\text { TUB1/URA3::GFP-TUB1 } \\
\text { LEU2::STU2p-STU2(R519A)- } \\
\text { 3V5:LEU2/LEU2::STU2p- } \\
\text { STU2(R519A)-3V5:LEU2 }\end{array}$ & This study \\
\hline RKY140 & $\begin{array}{l}\text { STU2-3HA-IAA7:kanMX/STU2-3HA- } \\
\text { IAA7:kanMX } \\
\text { HIS3::pDPD1-OsTIR1/HIS3::pDPD1- } \\
\text { OsTIR1 } \\
\text { TUB1/URA3::GFP-TUB1 } \\
\text { LEU2::STU2p-STU2(Acc2xbasic)- } \\
\text { 3V5:LEU2/LEU2::STU2p- } \\
\text { STU2(Acc2xbasic)-3V5:LEU2 }\end{array}$ & This study \\
\hline RKY132 & $\begin{array}{l}\text { STU2-3HA-IAA7:kanMX/STU2-3HA- } \\
\text { IAA7:kanMX } \\
\text { HIS3::pDPD1-OsTIR1/HIS3::pDPD1- } \\
\text { OsTIR1 } \\
\text { TUB1/URA3::GFP-TUB1 } \\
\text { LEU2::STU2p-STU2(R200A,R519A)- } \\
\text { 3V5:LEU2/ LEU2::STU2p- } \\
\text { STU2(R200A,R519A)-3V5:LEU2 }\end{array}$ & This study \\
\hline
\end{tabular}




\begin{tabular}{|l|l|l|}
\hline RKY139 & STU2-3HA-IAA7:kanMX/STU2-3HA- & This study \\
& IAA7:kanMX & \\
& HIS3::pDPD1-OsTIR1/HIS3::pDPD1- & \\
& OsTIR1 & \\
& TUB1/URA3::GFP-TUB1 & \\
& LEU2::STU2p-STU2(ACC)-3V5:LEU2/ & \\
& LEU2::STU2p-STU2(ACC)-3V5:LEU2 & \\
\hline
\end{tabular}

Table S2. Plasmids used in this study.

\begin{tabular}{|c|c|c|}
\hline Plasmid name & Genetic markers & Established in \\
\hline pASF125 & $\begin{array}{l}\text { For integration TUB1-GFP-TUB1-URA3 } \\
\text { at URA3 }\end{array}$ & (Straight et al. 1997) \\
\hline pGM25 & $\begin{array}{l}\text { For integration pZ4EV-SPC110(1-220)- } \\
\text { GCN4p1-GA-GFP-Lacl-ADE2 at ADE2 }\end{array}$ & (Lyon et al. 2016) \\
\hline pGM22 & LacOx256-TRP1 & (Lyon et al. 2016) \\
\hline pSB2232 & For integration STU2-3V5 at LEU2 & (Miller et al. 2016) \\
\hline pSB2306 & $\begin{array}{l}\text { For integration stu2(R200A)-3V5 at } \\
\text { LEU2 }\end{array}$ & (Miller et al. 2019) \\
\hline pSB2307 & $\begin{array}{l}\text { For integration stu2(R519A)-3V5 at } \\
\text { LEU2 }\end{array}$ & (Miller et al. 2019) \\
\hline pSB2308 & $\begin{array}{l}\text { For integration of stu2(R200A,R519A)- } \\
3 V 5 \text { at } L E U 2\end{array}$ & (Miller et al. 2019) \\
\hline pSB2261 & $\begin{array}{l}\text { For integration stu2( } \triangle 658- \\
\left.761:: G D G A G L^{\text {linker }}\right) \text { at } L E U 2\end{array}$ & (Miller et al. 2019) \\
\hline pSB3103 & $\begin{array}{l}\text { For integration stu2(2x_basic } 4658- \\
\left.761:: G D G A G L^{\text {linker }}\right) \text { at } L E U 2\end{array}$ & (Miller et al. 2019) \\
\hline
\end{tabular}




\section{Results and Discussion}

$\underline{\text { Spc110c }(T) \text { recruits the } \gamma \text {-tubulin complex and forms MT arrays in vivo }}$

Temporally controlled by an inducible promoter, the $\operatorname{Spc} 110 \mathrm{c}(\mathrm{T})$ recruits the $\gamma$ tubulin complex to the DNA, rather than the SPB, through lac operator/lac repressor interactions (Figure 1). This provides an ectopic site of nucleation that is distinct from the SPB. Over a three-hour observation period, $68 \pm 2.1 \%$ cells form MT arrays at the SPB, while $54 \pm 3.7 \%$ of cells form ectopic MT arrays at the Spc110c(T). Arrays at the Spc110c(T) are composed of antiparallel MTs with capped minus ends, as revealed by electron tomography (Figure 1C). While Spc110c(T)-associated MT bundles are less organized than mitotic spindles, MT minus ends cluster. 
A Organization at the SPB

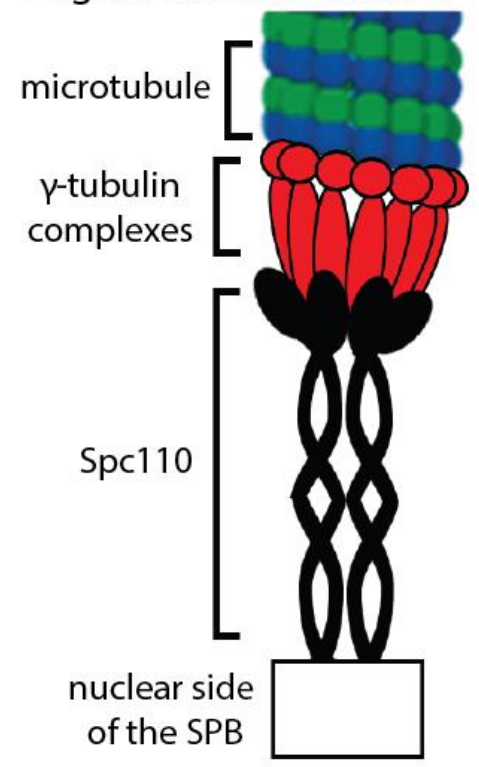

Organization at the Ectopic Nucleation Site

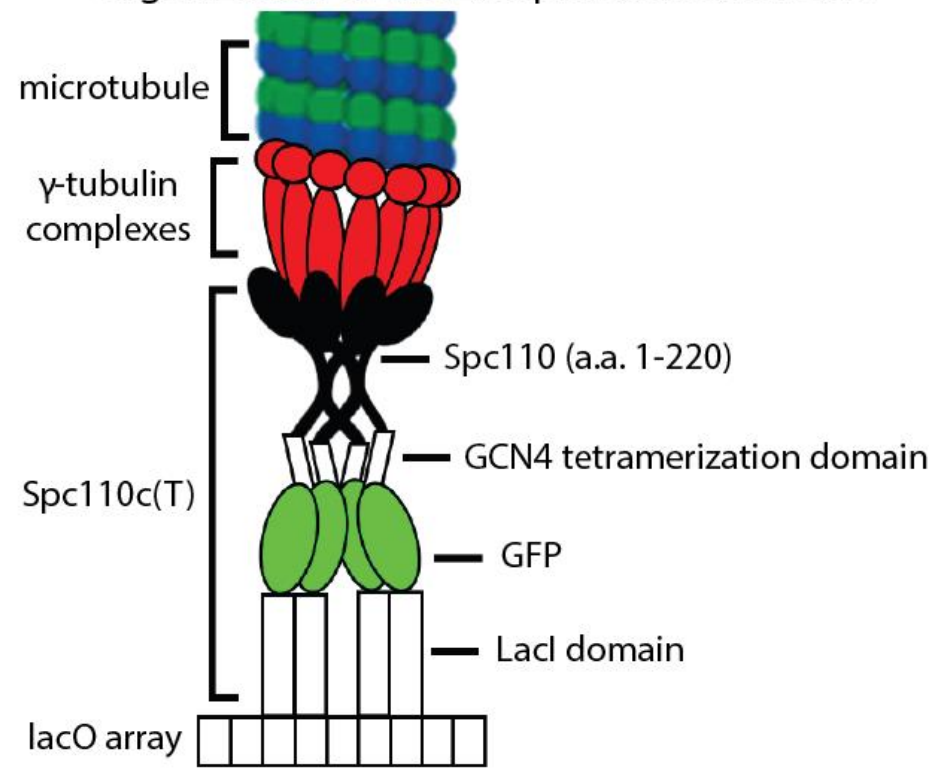

B

Representative cell expressing Spc110c(T), GFP-Tub1, and Spc97-mCherry mother daughter

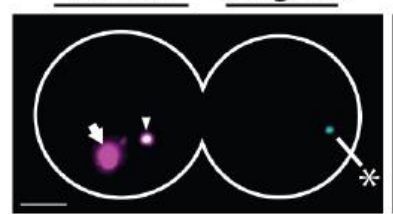

$\mathrm{t}=10 \mathrm{~min}$

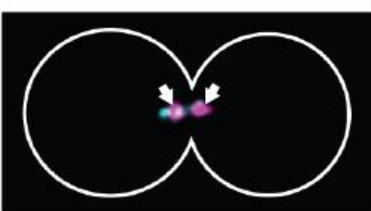

$\mathrm{t}=50 \mathrm{~min}$

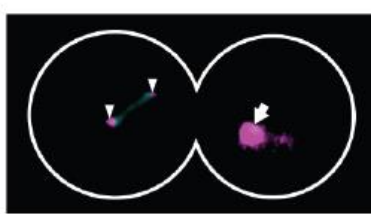

$\mathrm{t}=100 \mathrm{~min}$

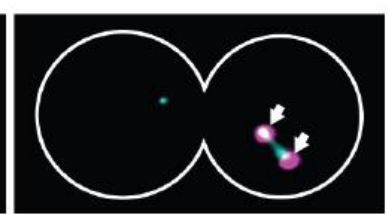

$\mathrm{t}=130 \mathrm{~min}$

SPBs form MT bundle ( $68 \pm 2.1 \%$ of cells)

Collapsed SPBs and ectopic nucleation sites ( $>90 \%$ of cells) ( $>90 \%$ of cells) and ectopic nucleation sites form MT bundle ( $54 \pm 3.7 \%$ of cells)

C

Cell 1

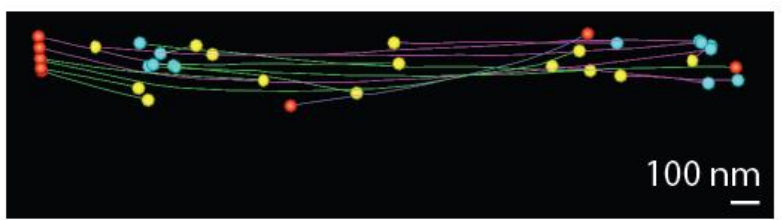

D Capped
minus end Flared

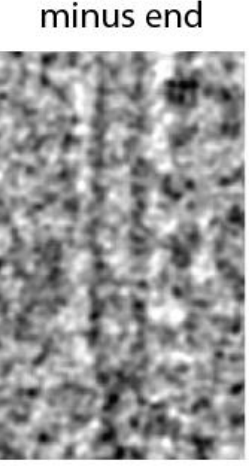
plus end

Cell 2

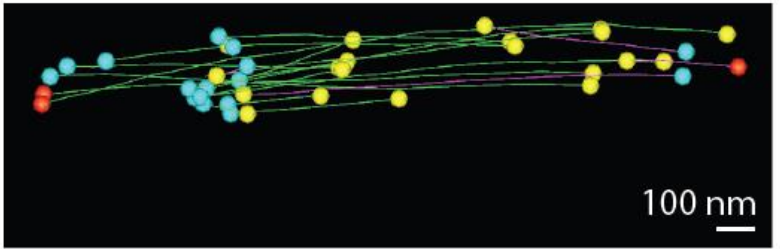

Cell 3
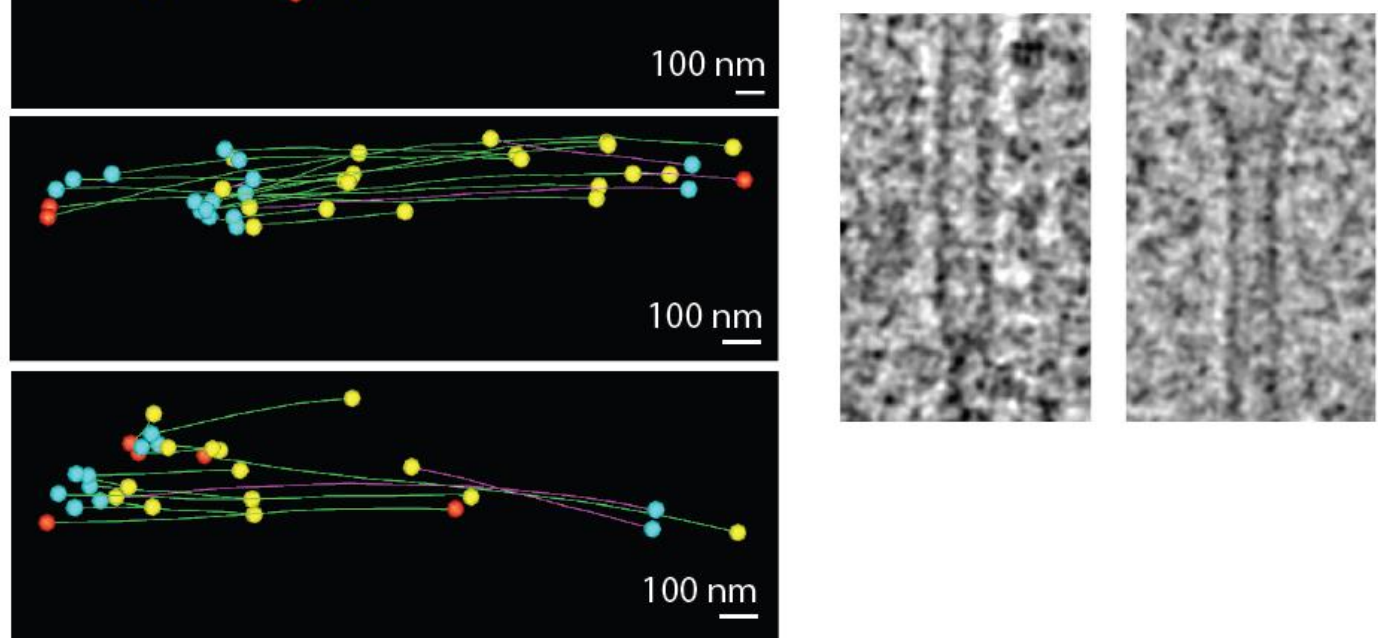
Figure 1. The $\gamma$-tubulin complex serves as a robust MT nucleation template at the wild-type SPB and the Spc110c(T).

(A) (Left) Full-length Spc110 dimerizes via a coiled coil domain and anchors $\gamma$-tubulin complexes to the nuclear side of the budding yeast SPB. (Right) The Spc110c(T) is comprised of the N-terminal 220 amino acids of Spc110, a tetramerization version of the GCN4 domain, a GFP tag, and a lac repressor domain which binds lacO arrays integrated into Chromosome XII.

(B) A representative timeline of events following nocodazole arrest, $\beta$-estradiol pulse induction of Spc110c(T), and nocodazole release (RKY52). At the earliest timepoints, SPBs are collapsed and the Spc110c(T) is colocalized with Spc97-mCherry. SPBs then move to the bud neck and into the daughter cell in $92 \pm 4.6 \%$ of cells. Following SPB movement to daughter, the Spc110c(T) forms MT arrays in the mother and the SPBs form spindles. $\gamma$-tubulin complexes are visualized using expression of Spc97$\mathrm{mCherry}$ (shown in magenta), and $\alpha \beta$-tubulin is visualized using expression of GFPTub1 (shown in cyan). Arrows denote SPBs and carets denote ectopically localized $\gamma$ tubulin complexes. Asterisk in left panel denotes a commonly observed phenomenon following Spc110c(T) expression-large GFP puncta in daughter cell that do not recruit Spc97-mCherry. Scale bar is $2 \mu \mathrm{M}$.

(C) Electron tomography of MT bundles associated with $\mathrm{Spc} 110 \mathrm{c}(\mathrm{T})$ revealed antiparallel MTs with clusters of capped minus ends (TVY11-123D). Capped minus ends shown in blue, flared plus ends shown in yellow, and MT ends out of volume shown in orange. Green MTs have minus end left of their plus end, and pink MTs have opposite polarity. Polarity of MTs was assessed by their visible ends.

(D) Representative capped minus end and flared plus end from electron tomography.

MAPs and motors are not required for localization of $\gamma$-tubulin complex to the

$\underline{\operatorname{Spc} 110 \mathrm{c}(T)}$

An initial, necessary step in MT nucleation is the recruitment of the $\gamma$-tubulin complex to Spc110 at the SPB. When cellular MTs are depolymerized in $15 \mu \mathrm{M}$ nocodazole, the $\operatorname{Spc} 110 \mathrm{c}(\mathrm{T})$ recruits the $\gamma$-tubulin complex, which is quantified as the percent of Spc110c(T) puncta that ectopically colocalize with Spc97-mCherry puncta (Figure 2). As described above, several of the candidate MAPs and motors interact with components of the SPB, so we asked whether the candidate proteins affected the ability of the $\mathrm{N}$-terminus of Spc110 to recruit or bind $\gamma$-tubulin complexes. We tested the effects 
of deletion of VIK1 and KIP3 and the effect of depletion of Stu2, Bim1, and Bik1 using an auxin-degradation system. We found that none of the MAPs or motors promote recruitment of the $\gamma$-tubulin complex to Spc110c(T) (Figure 2).

A

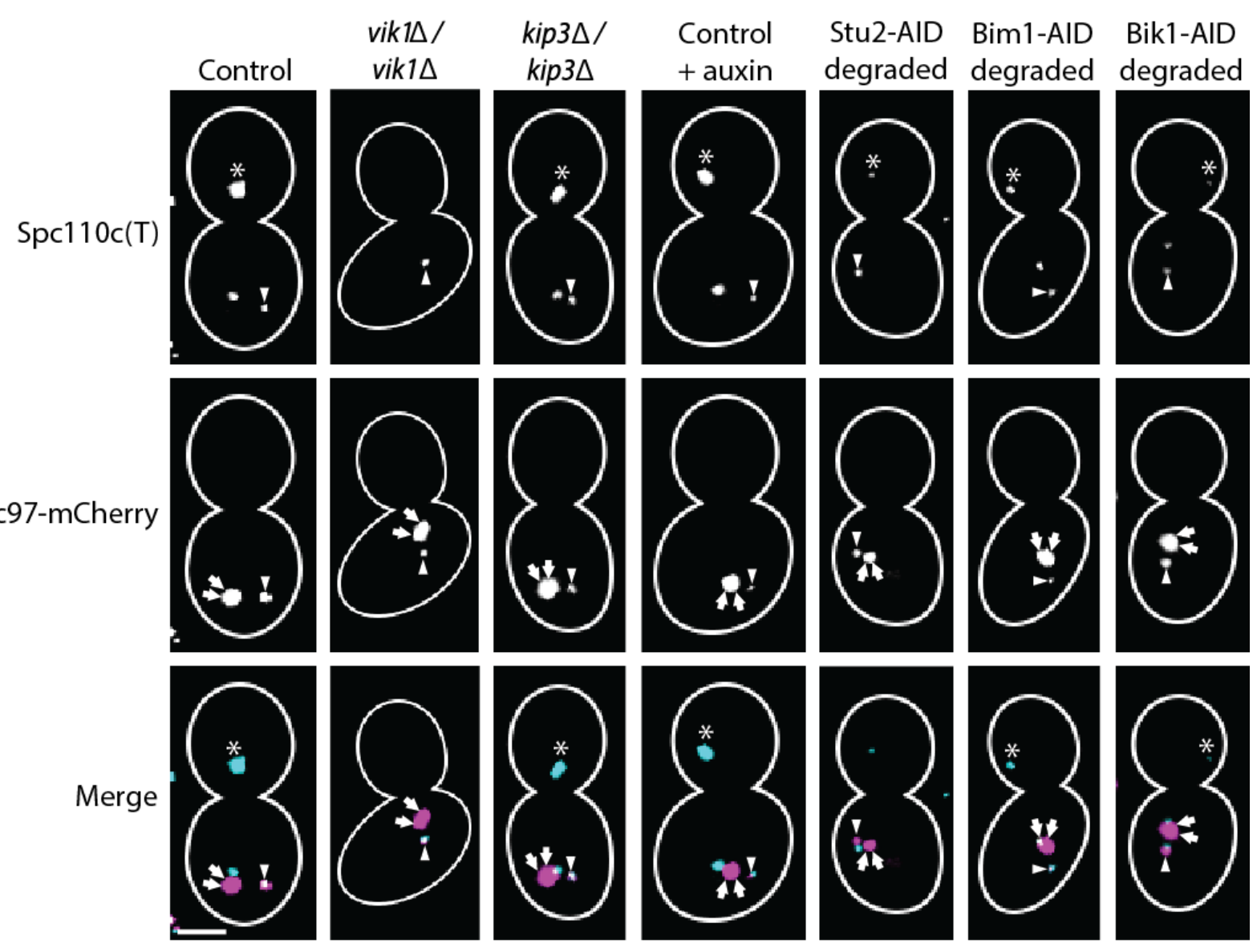

B

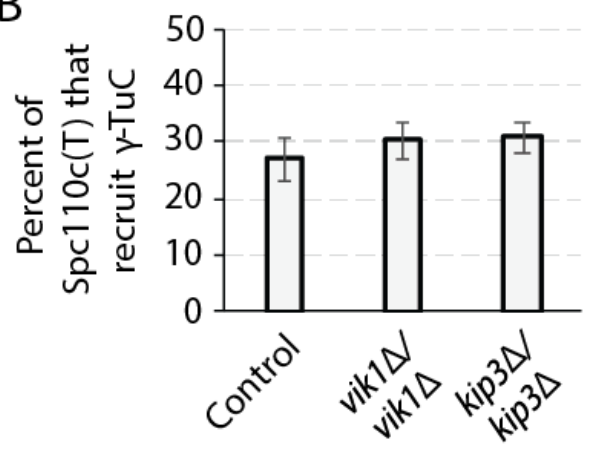

C

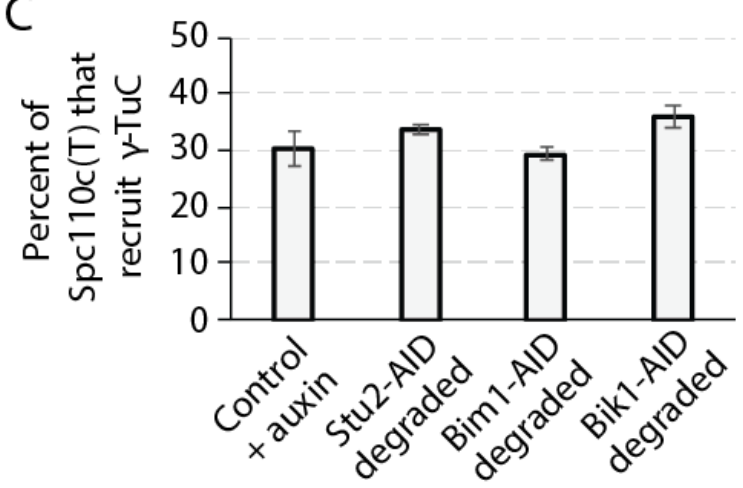

Figure 2. MAPs and motors are not required for $\gamma$-tubulin complex localization to the Spc110c(T). 
(A) Representative recruitment of $\gamma$-tubulin complex to Spc110c(T) in control (RKY44), vik14/vik14 (RKY174), kip34/kip34 (RKY56), and auxin-treated control, Stu2-AID (RKY14), Bim1-AID (RKY47), and Bik1-AID (RKY51) cells. $\gamma$-tubulin complexes are visualized using expression of Spc97-mCherry (shown in magenta). Arrows denote SPBs and carets denote ectopically localized $\gamma$-tubulin complexes. Asterisks denote GFP puncta in daughter cell that do not recruit Spc97-mCherry and are in the cytoplasm. Scale bar is $3 \mu \mathrm{M}$.

(B) Percent of cells in which the Spc110c(T) recruits the $\gamma$-tubulin complex is unaffected by deletion of Vik1 or Kip3. Values shown are mean \pm S.D. There were 2 biological replicates for all conditions and 234,316 , and 375 total cells for control,

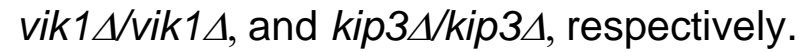

(C) Percent of cells in which the Spc110c(T) recruits the $\gamma$-tubulin complex is unaffected by degradation of Stu2, Bim1, or Bik1. Values shown are mean \pm S.D. There were 2 biological replicates for all conditions and 249, 389, 248, and 315 total cells for control plus auxin, Stu2-AID degraded, Bim1-AID degraded, and Bik1-AID degraded, respectively.

\section{$\underline{\text { Stu2, Bim1, Bik1, and Kip3 promote MT formation at the Spc110c(T) }}$}

Following nocodazole treatment and subsequent washout, the $\operatorname{Spc} 110 \mathrm{c}(\mathrm{T})$ promotes formation of ectopic MT arrays in $54 \pm 3.7 \%$ of cells, (32 $\pm 1.5 \%$ with auxin treatment) (Figure 3). We tested the role of Vik1, Kip3, Stu2, Bim1, and Bik1 in this phenomenon by quantifying the cumulative percent of cells forming MT arrays at both the SPBs and ectopic sites. We found that Vik1 was not required for MT array formation at either the SPB or the Spc110c(T) (Figures 3B and 3C). Strikingly, Stu2 and Bim1 are essential for MT formation from both the $\operatorname{Spc} 110 \mathrm{c}(\mathrm{T})$ and the SPB. No visible MTs form when either Stu2-AID or Bim1-AID is degraded (78\% and 99\% degradation, respectively) (Figures 3B and $3 \mathrm{C}$ ). 
Although not essential for MT array formation, both Kip3 and Bik1 each promote MT array formation at the Spc110c(T). For Kip3, MT formation is slightly delayed in $k i p 3 \Delta / k i p 3 \Delta$ cells at both the ectopic site and the SPB (Figure $3 \mathrm{C}$ ).

When Bik1-AID is degraded ( $82 \%$ degradation), MT formation is also delayed at the ectopic site, but by a unique mechanism. While degradation of Bik1-AID does not impact MT array formation between SPBs (Figure 3B), it does largely disrupt the movement of SPBs to the daughter (Figure 4). As seen in control cells, this movement of the SPB to the daughter enhances the formation of the ectopic MT bundle in the mother from $8.3 \%$ to $58 \%$ (Figure $4 \mathrm{~A}$ ). If the impact of Bik1 degradation on ectopic MT array formation is due to SPB localization, we would expect ectopic MT array formation to be approximately $11 \%$ (calculations shown in Materials and Methods). As the observed value is $12 \pm 0.36 \%$, the reduction of ectopic MT formation following Bik-AID degradation is most likely indirect and largely a result of the effect on SPB positioning. A role for Bik1 in SPB positioning has previously been observed (Carvalho et al. 2004; Caudron et al. 2008; McNally 2013). 
A
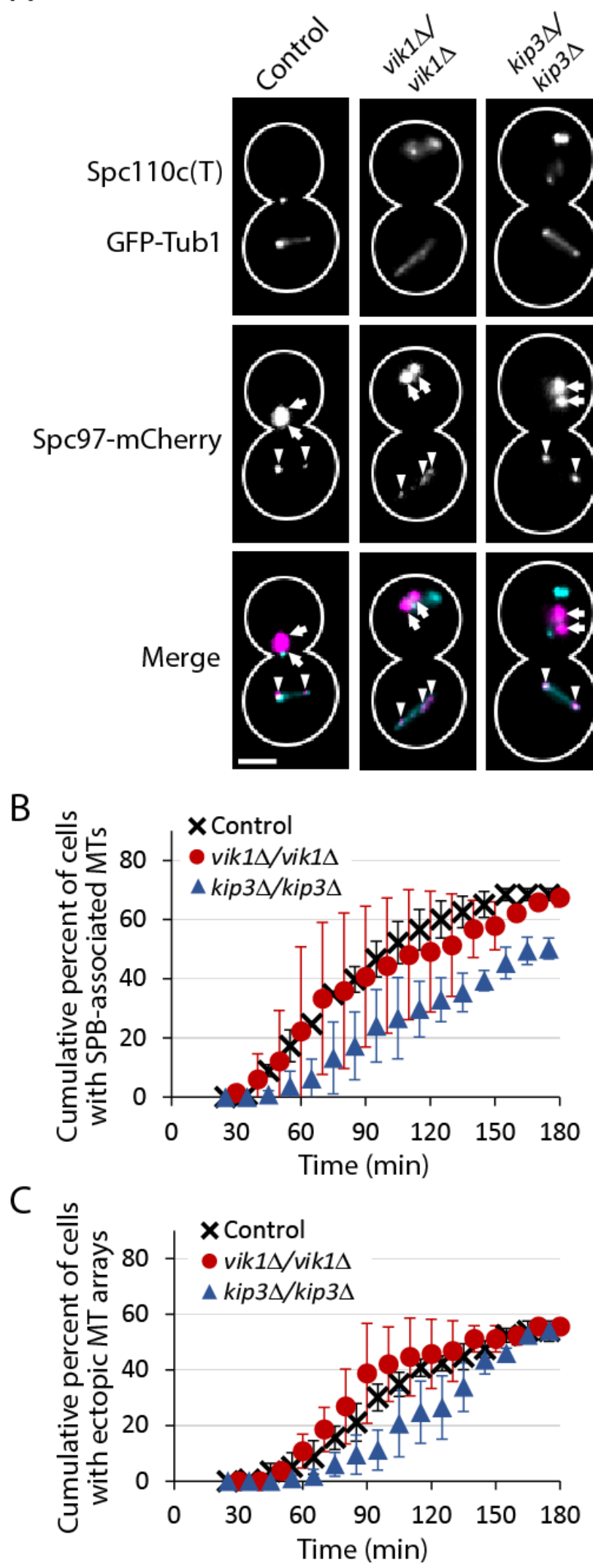
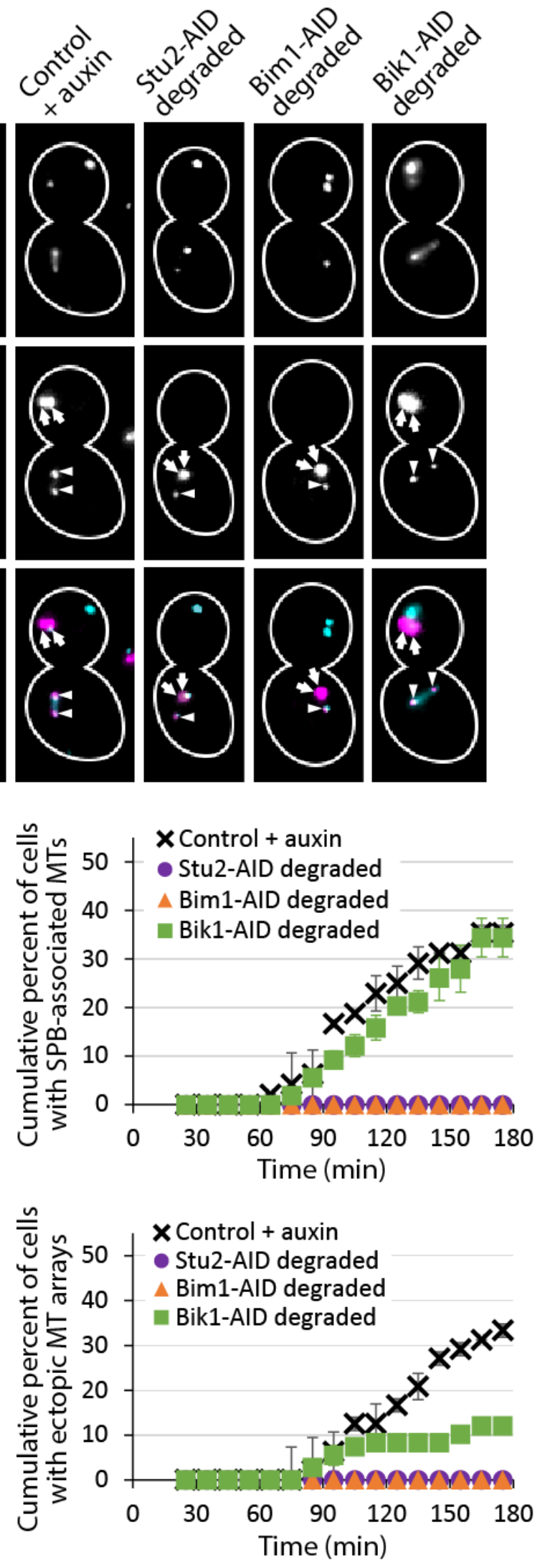
Figure 3. Stu2, Bim1, Bik1, and Kip3 promote MT array formation from the Spc110c(T).

(A) Representative MT array formation from the Spc110c(T) in control (RKY52),

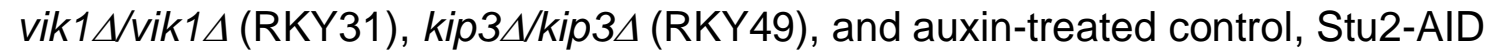
(RKY7), Bim1-AID (RKY46), and Bik1-AID (RKY36) strains. $\gamma$-tubulin complexes are visualized using expression of Spc97-mCherry (shown in magenta) and $\alpha \beta$-tubulin is visualized using expression of GFP-Tub1 (shown in cyan). Arrows denote SPBs and carets denote ectopically localized $\gamma$-tubulin complexes. Asterisks denote GFP puncta in daughter cell that do not recruit Spc97-mCherry. Scale bar is $3 \mu \mathrm{M}$.

(B) Cumulative percent of cells over time in which SPBs form spindles. Values shown are mean \pm S.D. There were $\geq 2$ biological replicates and 157, 108, and 120 total

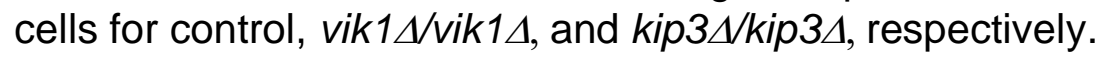

(C) Cumulative percent of cells over time in which Spc110c(T)s form MT arrays. Values shown are mean \pm S.D. There were 2 biological replicates for all conditions and 204, 173, 155, and 108 total cells for control plus auxin, Stu2-AID degraded, Bim1-AID degraded, and Bik1-AID degraded, respectively.
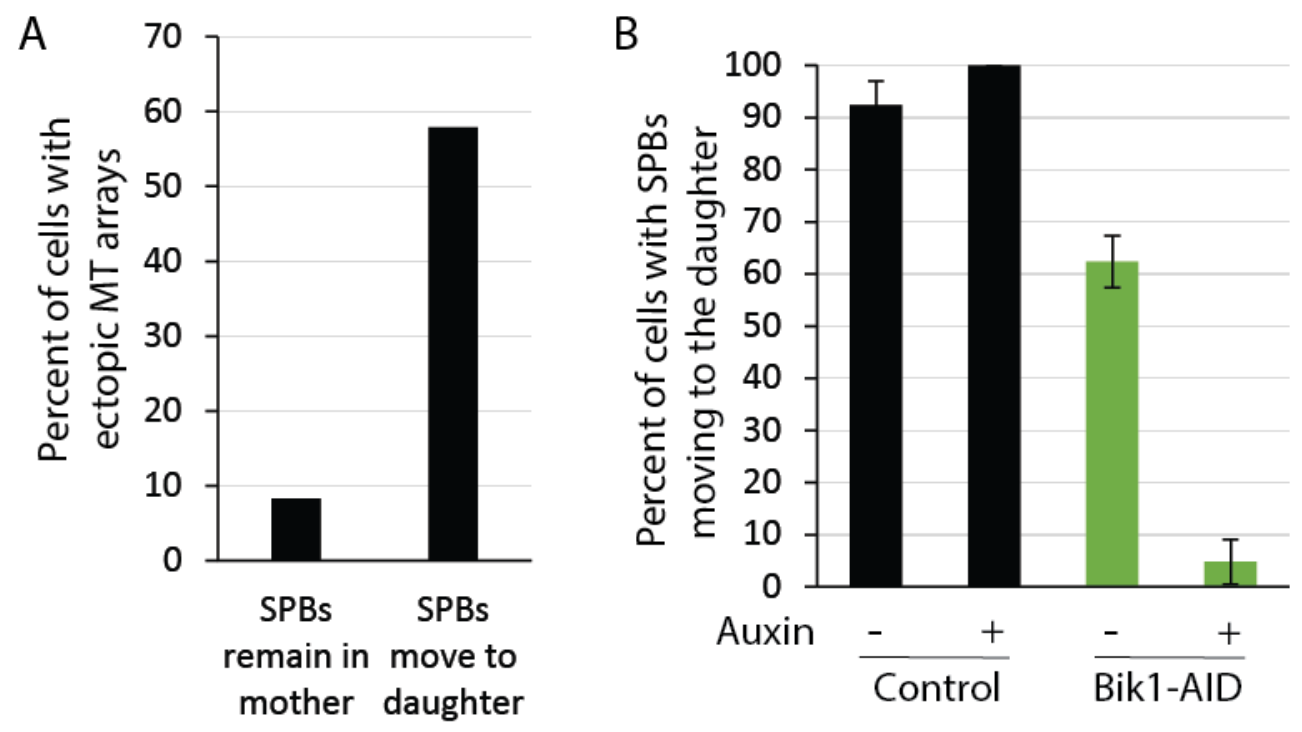

Figure 4. Bik1 indirectly promotes MT nucleation at the Spc110 chimera.

(A) SPB movement to daughter cells correlates positively with $\mathrm{Spc} 110 \mathrm{c}(\mathrm{T}) \mathrm{MT}$ formation. During the examination of ectopic MT formation in RKY52, cells were scored for the position of the SPB in the budded cells.

(B) Expression of Bik1-AID and depletion of Bik1-AID (RKY36) results in decreased SPB movement to daughter cells. Values shown are mean \pm S.D. There were $\geq 2$ biological replicates and 157, 204, 100, and 108 total cells, for control, control plus auxin, Bik1-AID, and Bik1-AID plus auxin, respectively. 
The ability of the $\operatorname{Spc} 110 \mathrm{c}(\mathrm{T})$ to form MT arrays correlates with the polymerase activity of Stu2

Recent work from our lab showed that the MT polymerization rate in vitro of XMAP215, the $X$. laevis homolog of Stu2, correlates with its ability to nucleate MTs in vitro. We asked whether previously published MT polymerization rates of Stu2 mutants in vitro would correlate with their promotion of MT array formation at the $\operatorname{Spc} 110 \mathrm{c}(\mathrm{T})$.

Stu2 polymerase activity can be modulated by inhibiting either its ability to bind free tubulin via TOG domains, to localize to the MT lattice via the basic MT binding domain, or to dimerize via the coiled coil domain. One study has quantified the polymerase activity of various Stu2 mutants, placing them into three categories: wildtype polymerase activity, moderate polymerase activity, or no activity (Figure 5A, data from (Geyer et al. 2018).

As shown above, Stu2 is required for MT formation following nocodazole treatment (Figure 3). When Stu2-AID is degraded, MT array formation is rescued through constitutive expression of Stu2 at an exogenous locus (Figure 5). We utilized this system to compare Stu2 mutants with different polymerase activities. Stu2-AID at its native locus was degraded during $\operatorname{Spc} 110 \mathrm{c}(\mathrm{T})$ expression, and Stu2 mutants were expressed constitutively at LEU2. As hypothesized, Stu2 polymerase activity in vitro correlated with MT formation activity (Figures $5 \mathrm{C}$ and $5 \mathrm{D}$ ). 
bioRxiv preprint doi: https://doi.org/10.1101/2021.01.12.426450; this version posted January 13,2021 . The copyright holder for this preprint (which was not certified by peer review) is the author/funder, who has granted bioRxiv a license to display the preprint in perpetuity. It is made available under aCC-BY-NC-ND 4.0 International license.

A Best polymerase
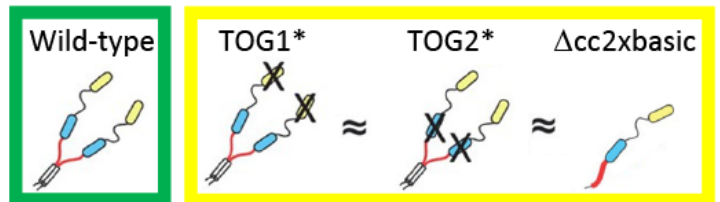

No polymerase activity

B

Stu2-AID degraded, Stu2 mutant expressed at LEU2

$\operatorname{Spc} 110 \mathrm{c}(\mathrm{T})$

GFP-Tub1
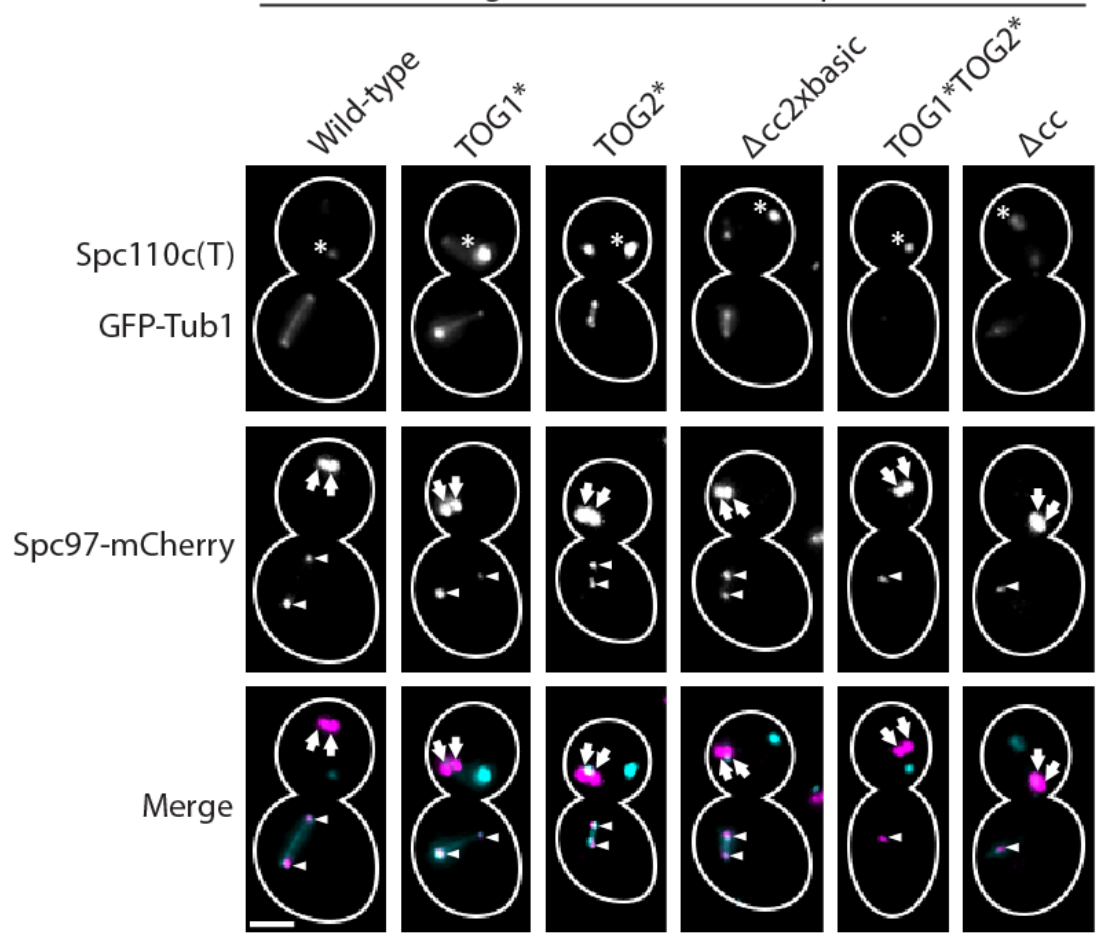

C

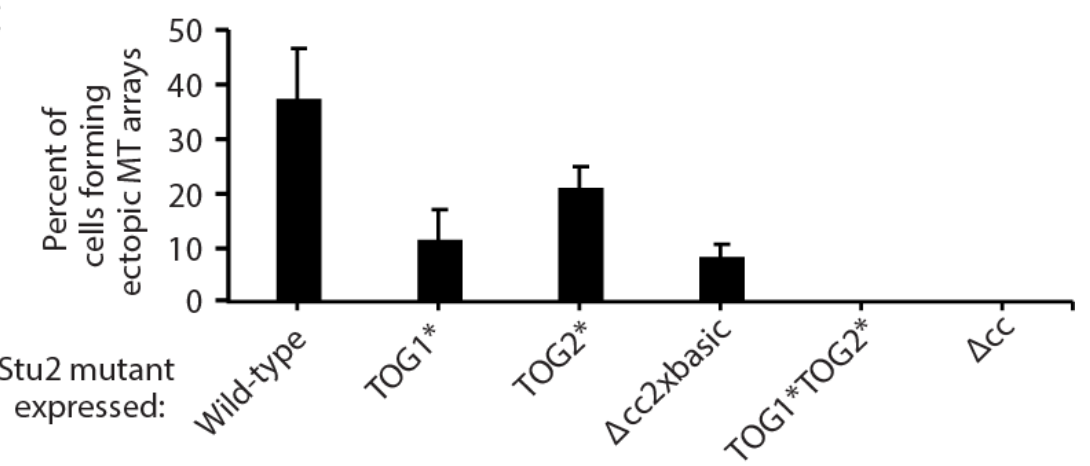

D

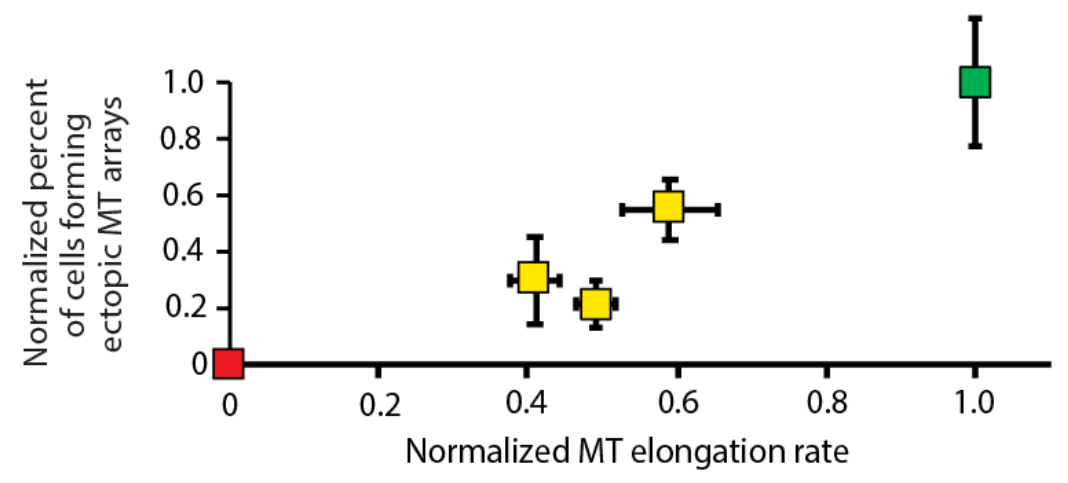


Figure 5. Stu2 promotion of MT nucleation at the $\operatorname{Spc110c(T)}$ correlates with its reported polymerase activity in vitro.

(A) Stu2 mutants used in this study fall into three distinct categories of polymerase activity, as quantified by (Geyer et al. 2018).

(B) Representative MT array formation at the Spc110c(T) when wild-type Stu2 (RKY122), Stu2 TOG1* (RKY126), Stu2 TOG2* (RKY133), Stu2 $\Delta$ cc 2xbasic (RKY140), Stu2 TOG1* TOG2* (RKY132), and Stu2 $\triangle \mathrm{Cc}$ (RKY139) are expressed. $\gamma$-tubulin complexes are visualized with Spc97-mCherry (shown in magenta) and tubulin is visualized with GFP-Tub1 (shown in cyan). Arrows denote SPBs and carets denote ectopically localized $\gamma$-tubulin complexes.

(C) Percent of cells in which Spc110c(T) forms a MT array over a three-hour period following nocodazole washout. Values are mean \pm S.D. There were 2 biological replicates for all conditions and 101,135, 157, 71, 138, and 154 total cells for Stu2, Stu2 TOG1*, Stu2 TOG2*, Stu2 $\triangle \mathrm{cc} 2$ xbasic, Stu2 TOG1* TOG2*, and Stu2 $\Delta \mathrm{cc}$, respectively.

(D) Percent of cells in which Spc110c(T) forms a MT array plotted against MT elongation rate, where each is normalized to the activity of wild-type Stu2. Data represents the results of Figure 5C and the published results of (Geyer et al. 2018). Colors correspond to color of boxes surrounding the depictions of the three classes of mutants in $(A)$.

$\underline{\text { Kinesin-14 motor complex protein Vik1 promotes focusing of MT minus ends }}$

While Vik1 was not required for ectopic recruitment of the $\gamma$-tubulin complex to Spc110c(T), nor ectopic MT array formation, deletion of VIK1 did result in decreased MT minus end clustering within the ectopic microtubule array (Figure 6). We quantified this phenotype by measuring Spc97-mCherry intensity along Spc110c(T)-associated MT arrays (Figure 6B). Importantly, the total Spc97-mCherry intensity along Spc110c(T)-associated MT arrays is the same in control cells as in vik1 $1 /$ vik1 1 cells (Figure 6C). However, in control cells, Spc97-mCherry usually localizes to two bright puncta at the end of ectopic MT arrays (73\% of cells, Figure 6D). In contrast, Spc97mCherry is more evenly distributed along $\operatorname{Spc} 110 \mathrm{c}(\mathrm{T})$-associated MT arrays in 
vik1 $1 /$ vik1 $1 \Delta$ cells. This phenotype was quantified using the root mean squared error (RMSE) (Equation 1):

$$
R M S E=\sqrt{\frac{1}{n} \sum_{i=1}^{n}\left(y_{i}-\hat{y}_{i}\right)^{2}}
$$

where $n=$ number of Spc110c(T) MT bundles measured, $y_{i}=$ Spc97-mCherry intensity at a given point within the bundle, and $\hat{y}_{i}=$ mean Spc97-mCherry intensity across the MT bundles. When $\gamma$-tubulin complexes cluster, the Spc97-mCherry intensity at any given point will be brighter and thus further from the mean of the Spc97-mCherry intensity along the MT bundle. Using this measure, Vik1 significantly promotes MT minus end clustering ( $p<0.001$, Figure $6 E)$. 
A

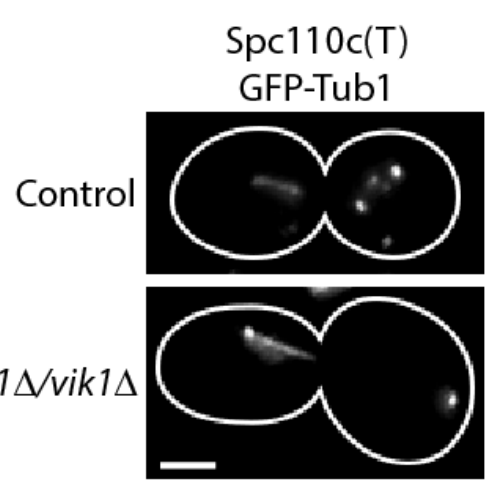

B

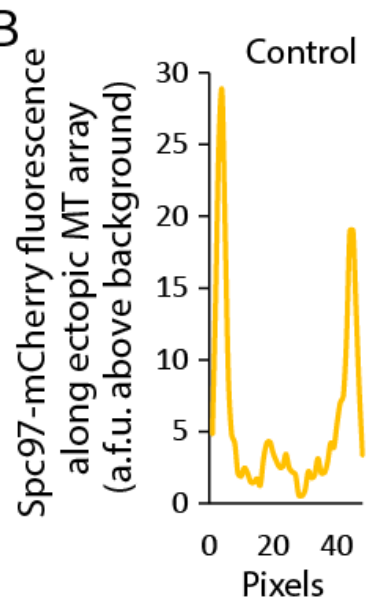

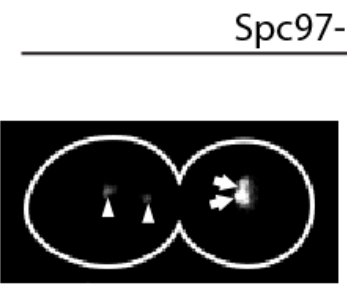

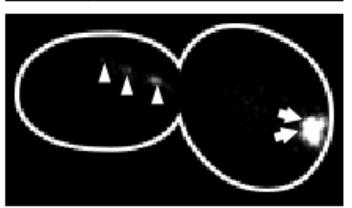

C

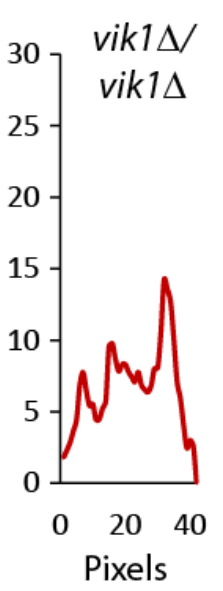

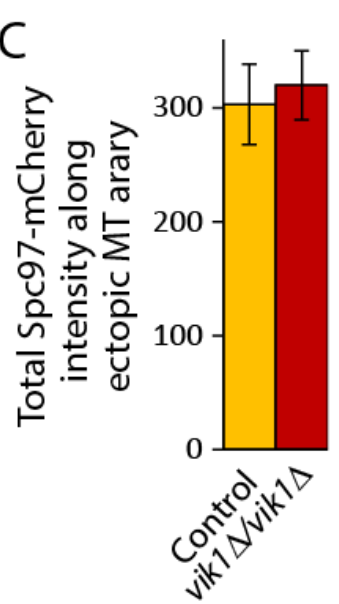

Merge

Spc97-mCherry intensity scaled for visualization
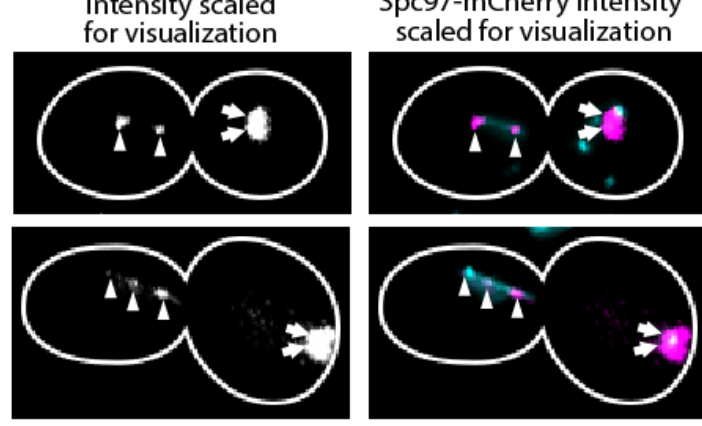

D Number of Spc97-mCherry peaks along ectopic MT array $\square 1 \square 2 \square 3 \square 4 \square 5$

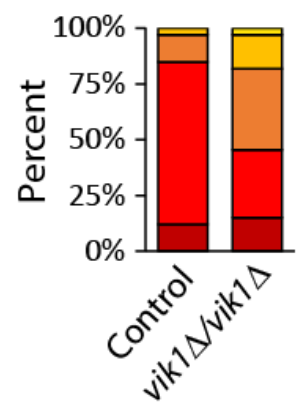

Figure 6. Vik1 significantly promotes $\gamma$-tubulin complex clustering.

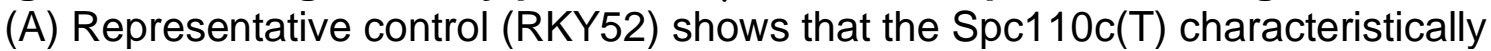
forms MT bundles with distinct $\gamma$-tubulin complex puncta at each end (top), and in $\Delta$ vik1/4vik1 (RKY31), the Spc110c(T) forms MT arrays with disperse $\gamma$-tubulin complexes along the array.

(B) Representative line scan of the average Spc97-mCherry intensity at a given pixel along the length of a 10-pixel wide line scan of the $\operatorname{Spc} 110 \mathrm{c}(\mathrm{T})$ associated MT array in control (left) and $\Delta v i k 1 / \Delta v i k 1$ cells (right). Data plotted is the sum intensity above background fluorescence across a 10-pixel line.

(C) Average total Spc97-mCherry intensity along Spc110c(T)-associated MT arrays.

(D) Number of Spc97-mCherry peaks along ectopic MT arrays differs between control cells and $\Delta v i k 1 / \Delta v i k 1$ cells.

(E) Average RMSE of Spc97-mCherry distribution along line scans of Spc110c(T)associated MT bundles. Data plotted are means \pm S.D. (control $\mathrm{N}=28, \operatorname{vik} 1 \Delta \mathrm{N}=$ 33). Asterisk denotes significance as measured by t-test, $p<0.001$.

The Spc110c(T) provides a unique system to analyze the roles of MAPs and motors whose roles have been obscured in the context of the resilient bipolar spindle. Here we report previously undetected roles for Stu2, Bim1, Bik1, Kip3 and Vik1 in the formation of a bipolar MT array in the nucleus. 
The formation of a bipolar array at the $\operatorname{Spc} 110 \mathrm{c}(\mathrm{T})$ can be divided into a number of steps, beginning with the recruitment of the $\gamma$-tubulin complex to the $\operatorname{Spc} 110 \mathrm{c}(\mathrm{T})$. Of the MAPs and motors included in this study, we found that none affected the ability of the $\operatorname{Spc} 110 \mathrm{c}(\mathrm{T})$ to recruit the $\gamma$-tubulin complex. The ability of the $\operatorname{Spc} 110 \mathrm{c}(\mathrm{T})$ to recruit might be strictly due to upstream regulation, such as post-translational modifications and/or oligomerization states of the nucleation machinery (Lyon et al. 2016).

The Spc110c(T) also revealed previously unreported roles in MT nucleation for Stu2, Bim1, Bik1, and Kip3 in budding yeast nuclei. Bim1 and Kip3 join their homologs as promoters of MT nucleation (Vitre et al. 2008; West and Mclntosh 2008; Erent et al. 2012). Bik1 becomes the first CLIP170 homolog to be implicated in MT nucleation, but due to its role in SPB positioning, the delay in MT formation at the Spc110c(T) may be an indirect consequence of SPB localization. Stu2, which localizes to the nucleus and the cytoplasm, has now been shown to promote MT nucleation in both compartments (Gunzelmann et al. 2018). Further, the polymerase activity of Stu2 in vitro correlates well with its nucleation activity in vivo, which supports the theory that Stu2 homologs function to elongate nascent MTs during nucleation (King et al. 2020).

Finally, we report a role for Vik1 in MT minus end focusing. Kinesin-14 motor proteins have been implicated in spindle repair via MT minus end focusing in other eukaryotic cells (Endow and Komma 1998; Kwon et al. 2008; Lecland and Lüders 2014). However, this is the first evidence of this role for Vik1, suggesting a broadly conserved role for the kinesin-14 motor proteins.

\section{Acknowledgments}

We thank Matthew Miller for the plasmids to integrate Stu2 mutants. This study was supported by grants from the National Institutes of Health grant P01 GM1 05537 to Mark Winey, Trisha Davis, R35 GM130293 to Trisha Davis, and T32 GM007270 to Brianna Rose King. 


\section{Author contributions}

BRK designed and performed research, analyzed the data, and wrote the manuscript. JBM performed tomography experiments. TV performed research. MW contributed experimental design, supervised research and edited the manuscript. EGM contributed experimental design and edited the manuscript. TND contributed experimental design, analyzed data, supervised the research, and helped write the manuscript.

\section{Conflict of interests}

The authors declare that they have no conflict of interest. 


\section{References}

Blake-Hodek, K., L. Cassimeris and T. C. Huffaker, 2010 Regulation of microtubule dynamics by Bim1 and Bik1, the budding yeast members of the EB1 and CLIP170 families of plus-end tracking proteins. Molecular Biology of the Cell 21: 20132023.

Brouhard, G. J., J. H. Stear, T. L. Noetzel, J. Al-Bassam, K. Kinoshita et al., 2008 XMAP215 is a processive microtubule polymerase. Cell 132: 79-88.

Burke, D., D. Dawson and T. Stearns, 2000 Methods in Yeast Genetics: A Cold Spring Harbor Laboratory Course Manual. Cold Spring Harbor, USA: Cold Spring Harbor Laboratory Press.

Carvalho, P., M. L. Gupta, M. A. Hoyt and D. Pellman, 2004 Cell cycle control of kinesin-mediated transport of Bik1 (CLIP-170) regulates microtubule stability and dynein activation. Developmental Cell 6: 815-829.

Caudron, F., A. Andrieux, D. Job and C. Boscheron, 2008 A new role for kinesindirected transport of Bik1p (CLIP-170) in Saccharomyces cerevisiae. Journal of Cell Science 121: 1506-1513.

Cottingham, F. R., L. Gheber, D. L. Miller and M. A. Hoyt, 1999 Novel roles for Saccharomyces cerevisiae mitotic spindle motors. Journal of Cell Biology 147: 335-349.

Derr, N. D., B. S. Goodman, R. Jungmann, A. E. Leschziner, W. M. Shih et al., 2012 Tug-of-war in motor protein ensembles revealed with a programmable DNA origami scaffold. Science 338: 662-665.

DeZwaan, T. M., E. Ellingson, D. Pellman and D. M. Roof, 1997 Kinesin-related KIP3 of Saccharomyces cerevisiae is required for a distinct step in nuclear migration. Journal of Cell Biology 138: 1023-1040.

Endow, S. A., and D. J. Komma, 1998 Assembly and dynamics of an anastral:astral spindle: the meiosis II spindle of Drosophila oocytes. Journal of Cell Science 111: 2487-2495.

Erent, M., D. R. Drummond and R. A. Cross, 2012 S. pombe kinesins-8 promote both nucleation and catastrophe of microtubules. PLoS ONE 7.

Fukuda, Y., A. Luchniak, E. R. Murphy and M. L. Gupta, 2014 Spatial control of microtubule length and lifetime by opposing stabilizing and destabilizing functions of kinesin-8. Current Biology 24: 1826-1835.

Furuta, K. y., A. Furuta, Y. Y. Toyoshima, M. Amino, K. Oiwa et al., 2013 Measuring collective transport by defined numbers of processive and nonprocessive kinesin motors. Proceedings of the National Academy of Sciences of the United States of America 110: 501-506.

Gardner, M. K., M. Zanic, C. Gell, V. Bormuth and J. Howard, 2011 Depolymerizing kinesins Kip3 and MCAK shape cellular microtubule architecture by differential control of catastrophe. Cell 147: 1092-1103.

Geiser, J. R., E. J. Schott, T. J. Kingsbury, N. B. Cole, L. J. Totis et al., 1997 Saccharomyces cerevisiae genes required in the absence of the CIN8- encoded spindle motor act in functionally diverse mitotic pathways. Molecular Biology of the Cell 8: 1035-1050. 
Geyer, E. A., M. P. Miller, C. A. Brautigam, S. Biggins and L. M. Rice, 2018 Design principles of a microtubule polymerase. eLife 7.

Gietz, R. D., R. H. Schiestl, A. R. Willems and R. A. Woods, 1995 Studies on the transformation of intact yeast cells by the LiAc/SS-DNA/PEG procedure. Yeast 11: 355-360.

Greenland, K. B., H. Ding, M. Costanzo, C. Boone and T. N. Davis, 2010 Identification of Saccharomyces cerevisiae Spindle Pole Body Remodeling Factors. PLoS ONE 5: e15426-e15426.

Gunzelmann, J., D. Rüthnick, T. C. Lin, W. Zhang, A. Neuner et al., 2018 The microtubule polymerase Stu2 promotes oligomerization of the $\mathrm{Y}$-TuSC for cytoplasmic microtubule nucleation. eLife 7.

Gupta, M. L., P. Carvalho, D. M. Roof and D. Pellman, 2006 Plus end-specific depolymerase activity of Kip3, a kinesin-8 protein, explains its role in positioning the yeast mitotic spindle. Nature Cell Biology 8.

Hoyt, M. A., L. He, K. K. Loo and W. S. Saunders, 1992 Two Saccharomyces cerevisiae kinesin-related gene products required for mitotic spindle assembly. Journal of Cell Biology 118: 109-120.

King, B. R., M. Moritz, H. Kim, D. A. Agard, C. L. Asbury et al., 2020 XMAP215 and gamma-tubulin additively promote microtubule nucleation in purified solutions. Molecular Biology of the Cell.

Kremer, J. R., D. N. Mastronarde and J. R. Mclntosh, 1996 Computer visualization of three-dimensional image data using IMOD. Journal of Structural Biology 116: 7176.

Kwon, M., S. A. Godinho, N. S. Chandhok, N. J. Ganem, A. Azioune et al., 2008 Mechanisms to suppress multipolar divisions in cancer cells with extra centrosomes. Genes and Development 22: 2189-2203.

Lecland, N., and J. Lüders, 2014 The dynamics of microtubule minus ends in the human mitotic spindle. Nature Cell Biology 16.

Lyon, A. S., G. Morin, M. Moritz, K. C. B. Yabut, T. Vojnar et al., 2016 Higher-order oligomerization of Spc110p drives gamma-tubulin ring complex assembly. Molecular Biology of the Cell 27: 2245-2258.

Manning, B. D., J. G. Barrett, J. A. Wallace, H. Granok and M. Snyder, 1999 Differential regulation of the Kar3p kinesin-related protein by two associated proteins, Cik1p and Vik1p. Journal of Cell Biology 144: 1219-1233.

Mastronarde, D. N., 1997 Dual-axis tomography: an approach with alignment methods that preserve resolution. Journal of Structural Biology 120: 343-352.

McNally, F. J., 2013 Mechanisms of spindle positioning, pp. 131-140. The Rockefeller University Press.

Melbinger, A., L. Reese and E. Frey, 2012 Microtubule Length Regulation by Molecular Motors. Physical Review Letters 108.

Miller, M. P., C. L. Asbury and S. Biggins, 2016 A TOG Protein Confers Tension Sensitivity to Kinetochore-Microtubule Attachments. Cell 165: 1428-1439.

Miller, M. P., R. K. Evans, A. Zelter, E. A. Geyer, M. J. MacCoss et al., 2019 Kinetochore-associated Stu2 promotes chromosome biorientation in vivo. PLoS Genetics 15. 
Miller, R. K., S. C. Cheng and M. D. Rose, 2000 Bim1p/Yeb1p mediates the Kar9pdependent cortical attachment of cytoplasmic microtubules. Molecular Biology of the Cell 11: 2949-2959.

Miller, R. K., K. K. Heller, L. Frisèn, D. L. Wallack, D. Loayza et al., 1998 The kinesinrelated proteins, Kip2p and Kip3p, function differently in nuclear migration in yeast. Molecular Biology of the Cell 9: 2051-2068.

Newman, J. R., E. Wolf and P. S. Kim, 2000 A computationally directed screen identifying interacting coiled coils from Saccharomyces cerevisiae. Proceedings of the National Academy of Sciences of the United States of America 97: 1320313208.

O'Connell, M. J., P. B. Meluh, M. D. Rose and N. R. Morris, 1993 Suppression of the bimC4 mitotic spindle defect by deletion of klpA, a gene encoding a KAR3related kinesin-like protein in Aspergillus nidulans. Journal of Cell Biology 120: 153-162.

O'Toole, E. T., M. Winey, J. R. McIntosh and D. N. Mastronarde, 2002 Electron tomography of yeast cells. Methods in Enzymology 351: 81-95.

Olmsted, Z. T., A. G. Colliver, T. D. Riehlman and J. L. Paluh, 2014 Kinesin-14 and kinesin- 5 antagonistically regulate microtubule nucleation by $\mathrm{Y}$-TuRC in yeast and human cells. Nature Communications 5: 1-15.

Page, B. D., L. L. Satterwhite, M. D. Rose and M. Snyder, 1994 Localization of the Kar3 kinesin heavy chain-related protein requires the Cik1 interacting protein. Journal of Cell Biology 124: 507-519.

Popov, A. V., F. Severin and E. Karsenti, 2002 XMAP215 is required for the microtubule-nucleating activity of centrosomes. Current Biology 12: 1326-1330.

Reese, L., A. Melbinger and E. Frey, 2011 Crowding of molecular motors determines microtubule depolymerization. Biophysical Journal 101: 2190-2200.

Roof, D. M., P. B. Meluh and M. D. Rose, 1992 Kinesin-related proteins required for assembly of the mitotic spindle. Journal of Cell Biology 118: 95-108.

Saunders, W., V. Lengyel and M. A. Hoyt, 1997 Mitotic spindle function in Saccharomyces cerevisiae requires a balance between different types of kinesinrelated motors. Molecular Biology of the Cell 8: 1025-1033.

Shetty, A., N. I. Reim and F. Winston, 2019 Auxin-Inducible Degron System for Depletion of Proteins in Saccharomyces cerevisiae. Current Protocols in Molecular Biology 128.

Straight, A. F., W. F. Marshall, J. W. Sedat and A. W. Murray, 1997 Mitosis in Living Budding Yeast: Anaphase A But No Metaphase Plate. Science 277: 574-578.

Su, X., W. Qiu, M. L. Gupta, J. B. Pereira-Leal, S. L. Reck-Peterson et al., 2011 Mechanisms Underlying the Dual-Mode Regulation of Microtubule Dynamics by Kip3/Kinesin-8. Molecular Cell 43: 751-763.

Thawani, A., R. S. Kadzik and S. Petry, 2018 XMAP215 is a microtubule nucleation factor that functions synergistically with the $\mathrm{Y}$-tubulin ring complex. Nature Cell Biology 20: 575-585.

Thomas, B. J., and R. Rothstein, 1989 Elevated recombination rates in transcriptionally active DNA. Cell 56: 619-630. 
Tischer, C., D. Brunner and M. Dogterom, 2009 Force- and kinesin-8-dependent effects in the spatial regulation of fission yeast microtubule dynamics. Molecular Systems Biology 5: 250-250.

Usui, T., H. Maekawa, G. Pereira and E. Schiebel, 2003 The XMAP215 homologue Stu2 at yeast spindle pole bodies regulates microtubule dynamics and anchorage. EMBO Journal 22: 4779-4793.

Varga, V., J. Helenius, K. Tanaka, A. A. Hyman, T. U. Tanaka et al., 2006 Yeast kinesin-8 depolymerizes microtubules in a length-dependent manner. Nature Cell Biology 8.

Vitre, B., F. M. Coquelle, C. Heichette, C. Garnier, D. Chrétien et al., 2008 EB1 regulates microtubule dynamics and tubulin sheet closure in vitro. Nature Cell Biology 10: 415-421.

Wang, Y., X. Zhang, H. Zhang, Y. Lu, H. Huang et al., 2012 Coiled-coil networking shapes cell molecular machinery. Molecular Biology of the Cell 23: 3911-3922.

West, R. R., and J. R. Mclntosh, 2008 Novel interactions of fission yeast kinesin 8 revealed through in vivo expression of truncation alleles. Cell Motility and the Cytoskeleton 65: 626-640.

Wieczorek, M., S. Bechstedt, S. Chaaban and G. J. Brouhard, 2015 Microtubuleassociated proteins control the kinetics of microtubule nucleation. Nature Cell Biology 17: 907-918.

Wigge, P. A., O. N. Jensen, S. Holmes, S. Soues, M. Mann et al., 1998 Analysis of the Saccharomyces spindle pole by matrix-assisted laser desorption/ionization (MALDI) mass spectrometry. Journal of Cell Biology 141: 967-977. 